\title{
Erratum to: Number Rigidity in Superhomogeneous Random Point Fields
}

\author{
Subhro Ghosh ${ }^{1}$ - Joel Lebowitz ${ }^{2}$
}

Published online: 30 December 2016

C Springer Science+Business Media New York 2016

\section{Erratum to: J Stat Phys DOI 10.1007/s10955-016-1633-6}

In the online published version the NSF grant number for JLL is incorrect. This has been corrected with this erratum.

The correct statement is given below.

The NSF grant JLL should be DMR 1104501 instead of DMR 1104500.

The online version of the original article can be found under doi:10.1007/s10955-016-1633-6.

Subhro Ghosh

subhrowork@gmail.com

Joel Lebowitz

lebowitz@math.rutgers.edu

1 Department of ORFE, Princeton University, Princeton, NJ, USA

2 Department of Mathematics and Physics, Rutgers University, New Brunswick, NJ, USA 